\title{
Instability of $\mathrm{Ag}$ nanoparticles in $\mathrm{SiO}_{2}$ at ambient conditions
}

\author{
Matthias Hillenkamp
}

Giulia Di Domenicantonio

Olivier Eugster

\section{Christian Félix}

Institut de Physique des Nanostructures, École Polytechnique Fédérale de Lausanne CH-1015 Lausanne-EPFL, Switzerland

E-mail: matthias.hillenkamp@epfl.ch

\begin{abstract}
The room temperature stability of nanometer-sized silver clusters in silica matrices has been investigated by following the temporal evolution of their surface plasmon absorption. $\mathrm{Ag}$ clusters in $\mathrm{SiO}_{2}$ were prepared by either annealing silica samples doped with atomic silver or by co-deposition of pre-formed clusters of defined size. Clusters were found to be unstable at ambient conditions on a time scale of days to weeks, independent of preparation conditions. The disappearance of the plasmonic resonance is explained by successive oxidation of the clusters even inside the matrix.
\end{abstract}

PACS numbers: $36.40 . \mathrm{Vz}, 36.40 . \mathrm{Qv}, 78.66 . \mathrm{w}$

Submitted to: Nanotechnology 


\section{Introduction}

Composite materials of metal nanoclusters in quartz matrices have been drawing much attention in the last years owing to their potential applicability in optical and optoelectronical devices [1,2]. Their use in integrated optics [3] and waveguides [4] stems mainly from the large third order non-linear susceptibility due to the surface plasmon excitations of nanometric metal particles [5]. But also in basic research the plasmon absorption and its modelling following Mie theory have proven a versatile tool in understanding cluster size effects, specific quantum mechanical influences [6] and interface effects [7].

Recently it was shown that also very small silver clusters of a few atoms only have intriguing properties and that their fluorescence is not only an ideal tool for fundamental researchers to determine their atomic and electronic structure [8,9] but that it might also be exploited in applications [10].

The aim of our research is to find means to produce samples of well defined metal clusters stabilized in protective matrices and to study their properties $[11,12]$. In this article we present a series of feasibility studies on the generation of tailor-made samples of metal clusters embedded in co-evaporated silica matrices.

\section{Experiment}

Samples were prepared following the technique of 'cluster-assembled materials'. The experimental setup has been described elsewhere [11] and will only be sketched briefly. Atomic or cluster ions are generated in the gas phase in a magnetron cluster source following the design of Haberland et al. [13]. The ion beam is characterized and optimized by time-of-flight mass spectrometry (TOF-MS) and afterwards directed towards the deposition chamber. Here the ions are co-deposited fragmentation-free together with the $\mathrm{SiO}_{2}$ matrix onto synthetic fused silica substrates (Tetrasil or Spectrosil, St. Gobain Switzerland). A filament, shielded from the sample in order to avoid spurious deposition of tungsten atoms, provides thermal electrons in the vicinity of the substrate. As soon as a significant charge accumulates on the surface, electrons are attracted and thus avoid charging of the sample. The quartz matrix is evaporated by an electron beam evaporator in a different chamber separated by a liquid nitrogen cooled diaphragm. Sample thicknesses used here are between 200 and $3000 \mathrm{~nm}$, the molar Ag concentration was kept well below 1\% to prevent aggregation.

Cluster samples were generated in two ways: either by annealing and thus thermally assisted aggregation of atomic silver or by direct embedding of preformed clusters. The first, more common technique consists of co-deposition of silver atoms and the silica matrix, followed by thermal annealing for $\sim 1 \mathrm{~h}$ at $500^{\circ} \mathrm{C}$ in controlled atmosphere. While simple, this method suffers from large, a priori unknown cluster size distributions. The latter technique, on the other hand, allows a precise fabrication of monodispersed cluster samples by depositing clusters of well-defined and known size. No further 
annealing of these samples was performed.

Optical absorption and fluorescence spectra were measured ex-situ in air using a deuterium lamp and a spectrometer with a liquid-nitrogen-cooled CCD-camera. Matrix stoichiometry was verified by X-ray photoelectron spectroscopy (XPS) and found to be 1:2 for Si:O. Film thicknesses were determined with an alpha-step profilometer, no densification of the quartz films after annealing at $500^{\circ} \mathrm{C}$ was detectable.

\section{Results}

Absorption and fluorescence spectra of samples containing only atomic silver showed no signal (figure 1). The absence of photoluminescence at $330 \mathrm{~nm}$ after excitation at $228 \mathrm{~nm}$ implies that atomic silver is incorporated in pure $\mathrm{SiO}_{2}$ as $\mathrm{Ag}^{0}$. This was also confirmed by XPS and is in agreement with previous observations [14]. No other peaks attributed to dimers and larger clusters $[15,16]$ were found confirming the low mobility of $\mathrm{Ag}$ on the amorphous silica surface.

Several of the samples containing atomic silver were annealed for $\sim 1 \mathrm{~h}$ at $500^{\circ} \mathrm{C}$ in different atmospheres. Samples annealed in a reducing atmosphere of $\sim 800 \mathrm{mbar}$ $5 \% \mathrm{H}_{2} / 95 \% \mathrm{~N}_{2}$ showed a yellowish colour and a clear absorption near $3 \mathrm{eV}$ (figure 1) typical for the surface plasmon resonance of nanometric Ag clusters in silica matrices [17]. Samples annealed in either air or vacuum under otherwise identical conditions did not display any such absorption. Surprisingly this plasmon absorption peak was not stable over time, after three weeks at ambient conditions no more absorption was detectable and the sample had lost its colour. It was, however, possible to reactivate the plasmonic absorption by further annealing in the same reducing atmosphere. Annealing of the same sample in vacuum did not reactivate the plasmonic absorption. Consecutive measurements of the plasmon peak of both annealed and deposited cluster samples reveal a continuous displacement of the mean plasmon resonance frequency with time, as shown in figure 2 .

In order to further clarify the mechanism behind this room temperature instability, samples with narrow distributions of well defined, pre-formed clusters in silica were produced. Mean cluster sizes used were $\langle n\rangle=700,1200,2700$ and 3600 atoms/cluster (corresponding to a mean diameter between $2.8 \mathrm{~nm}$ and $4.9 \mathrm{~nm}$ ). All samples showed a plasmonic absorption after removing from vacuum and measuring at ambient conditions, as shown in figure 3. About one week later, this absorption had disappeared, too.

\section{Discussion}

First of all the observed sample ageing is somewhat surprising. Ageing of silica samples containing atomic silver prepared by sol-gel technique has previously been observed; on the time scale of several months initially transparent films developed a faint yellowish colour characteristic of plasmonic absorption [14]. The authors show that in their samples atomic silver accumulates at the film-substrate interface during preparation and 


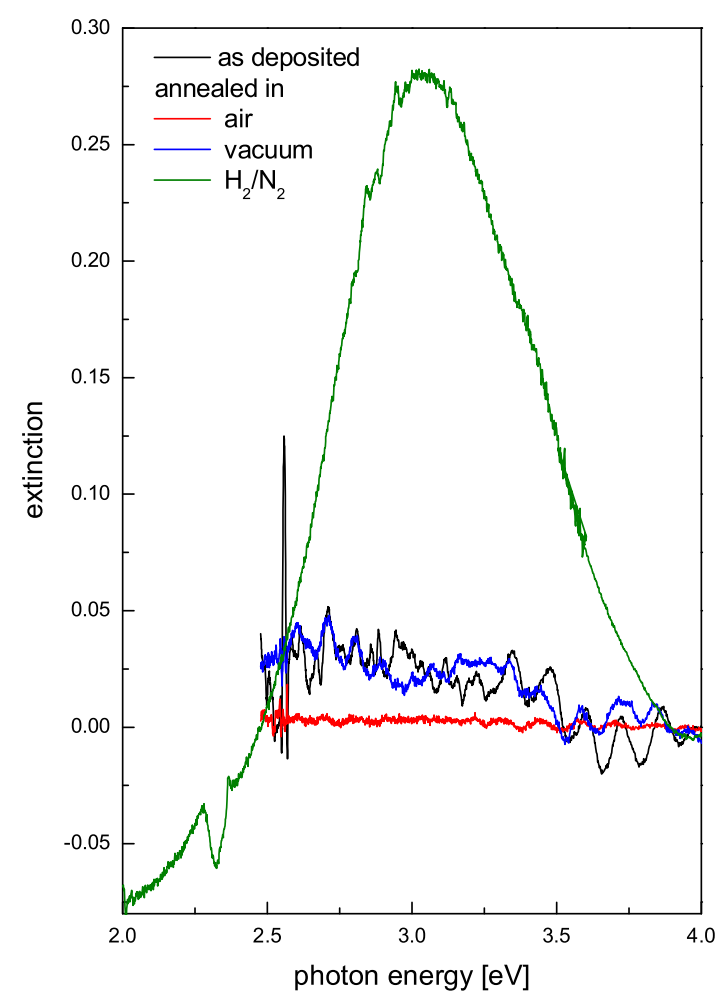

Figure 1. (colour online) Optical absorption spectra of silica samples doped with atomic silver before and after annealing in different atmospheres. Only samples annealed in reducing atmosphere show a plasmonic absorption. The small oscillations are interference artifacts due to reflections at the matrix interfaces.

slowly aggregates into larger clusters. This demonstrates that although the mobility of $\mathrm{Ag}$ atoms inside the quartz matrix is low at room temperature, atoms near an interface show an increased mobility. No ageing in the opposite direction, i.e. of the form of disappearing plasmonic signal has been reported in the literature to our knowledge.

The redox kinetics of silver nanoparticles in meso-porous silica matrices has been studied by several groups, showing e.g. that the critical temperature for complete oxidation is size dependent and lies around $500^{\circ} \mathrm{C}$ for $2 \mathrm{~nm}$ particles [18]. Other experiments demonstrated that in such meso-porous networks (porosity 50\%) it is possible to run several redox cycles of Ag clusters by annealing in different atmospheres, thereby quenching and reactivating the plasmonic absorption [19]. The authors show a comparison with denser matrices such as ion-exchanged soda-lime glasses, where only a minor reduction of the plasmon is observed.

The work of Celep et al. [20] is the only study of oxidation influences on the preparation of dielectric matrices with pre-formed clusters we know of, in this case $\mathrm{Cu}_{n}$ in alumina. They show that only deposition at elevated temperatures $\left(400^{\circ} \mathrm{C}\right.$ as opposed to room temperature) allows the fabrication of unoxidized clusters with the typical plasmon 
resonance.

On a qualitative level the optical absorption properties of both our annealed atomic samples as well as those containing preformed clusters are comparable. It can thus be concluded that the observed room temperature instability is independent of cluster properties and production mechanism. The fact that a reducing atmosphere is needed to regenerate the clusters' metallic character clearly shows that the observed ageing is not due to a physical loss of structural integrity that should be reversible by increasing the atomic mobility at high temperatures but rather due to a chemical reaction.

Since a continuous red-shift of the plasmon resonance peak has been observed for Ag clusters deposited on a quartz surface and exposed to molecular oxygen [7], we can assume cluster oxidation to be responsible for the shift in our samples, too. In the case of clusters on a surface, however, only a slight decrease in peak intensity is reported, the plasmon resonance does not disappear. The authors interpret this as a weak surface oxidation that leaves the surface passivated and prevents further volume oxidation.

The observed cluster instability in our samples can thus be explained by successive oxidation of the clusters. Since the intrinsic oxygen at the cluster-matrix interface is

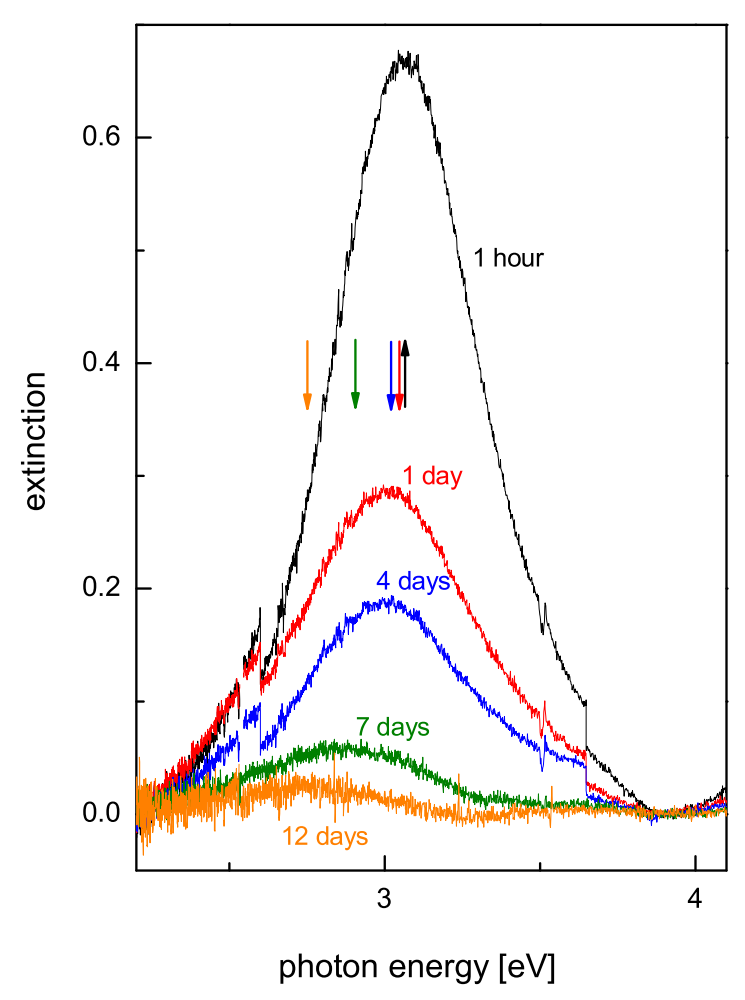

Figure 2. (colour online) Absorption spectra of a sample containing clusters with $\langle n\rangle=3600$ atoms/cluster, measured on consecutive days. The red-shift of the plasmon absorption can be attributed to oxidation of the Ag clusters and is visualized by the arrows indicating the mean absorption energy. 


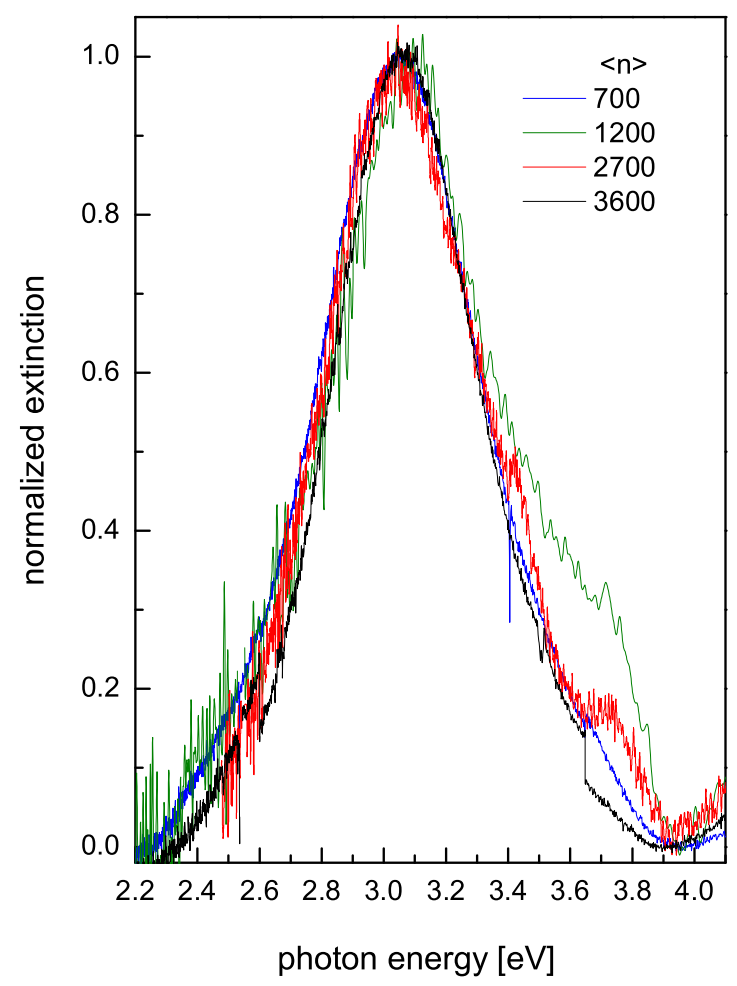

Figure 3. (colour online) Absorption spectra for different cluster sizes co-deposited with the silica matrix. All samples show comparable plasmonic absorption signals.

not enough to fully oxidize the clusters, additional oxygen or water must diffuse into the matrix network where an oxygen-assisted exchange of atoms between the cluster and the surrounding matrix seems most probable. This mechanism takes place on a timescale of days and explains both the shift as well as the signal decrease of the plasmon resonance. A short discussion of the structure of our evaporated silica films is indicated at this moment. Silica films are amorphous, three-dimensional networks of largely intact vertex-sharing $\mathrm{SiO}_{4}$ tetrahedra [21]. Sol-gel prepared silica matrices are usually porous, depending on the production conditions and they can be densified through pore closure by annealing. For thin films this densification is already significant at temperatures around $500^{\circ} \mathrm{C}[22]$. The mean porosity of such films can be estimated by comparing the refractive index with that of fused silica. We measured the refractive index of freshly prepared undoped films by ellipsometry in the visible range to 1.41 (as compared to 1.46 for fused silica). We derive a mean porosity of $\leq 10 \%$, which is significantly lower than for e.g. the alumina films used in ref. [20]. Consequently no silica defects other than a small fluorescence signal from the substrate attributed to an oxygen deficiency center (ODC(II) [23]) were optically detectable. Also no densification after annealing was measurable.

This macroscopic estimate nevertheless has to be taken cautiously, since obliquely 
evaporated silica films have been shown to grow in a columnar structure (with an index of refraction of 1.25) [24]. Although we were not able to exclude this for our matrices grown at $45^{\circ}$, the considerably higher index of refraction indicates a denser structure. On the other hand an increased porosity has to be assumed for samples containing preformed clusters, since the clusters are only weakly deformed during soft-landing and thus shade off a certain area. While it seems possible that atoms accumulate near pore or column edges during annealing and might thus be more exposed to oxygen, this is not conceivable for preformed clusters. They would need to diffuse as a whole on the silica surface before being covered, an improbable scenario especially when bearing in mind the high density of surface defects, the comparably low temperature and the low mobility of atoms at room temperature (see above). We thus conclude that oxygen or water penetrates even dense silica matrices on a scale of days, not only through pores but even through the matrix network.

We finally want to address the lack of size dependence of the plasmon resonance in figure3. The mean energy and width of nanometric noble metal particles are known to sensitively depend on several parameters. Besides the evident elemental choice of metal and matrix, cluster size and local environment (oxygen content, porosity etc.) can also influence the optical properties [6]. Ag clusters plasmons are known to display a shift in energy as well as an increasing peak width with decreasing size when measured in the gas phase or in matrices such as Argon or annealed ion-exchanged glasses [6]. Preformed Ag clusters, co-deposited in alumina matrices, however, show no clear size dependence in neither position nor width of the plasmon resonance $[25,26]$. The authors explain this as a consequence of competing effects, i.e. the reduction of the mean free path of free electrons inside the cluster and spill-out of electronic density, all of which are sensitively influenced by the surrounding matrix, i.e. both its electronic structure as well its exact geometry (porosity). Apparently all size effects are quenched in our evaporated silica matrices as well, at least in the size range studied.

This is emphasized by the fact that the plasmon absorptions for all samples studied display comparable consecutive time-dependent red-shifts, implying that not the cluster size but rather the chemical environment (a metal-oxide layer) is responsible for any apparent size effect.

\section{Conclusions}

In this work we have investigated the room temperature stability of silver clusters embedded in quartz matrices prepared by different methods. The observation of the plasmon resonance absorption directly shows whether or not a metallic particle of at least several hundreds of atoms is present. Clusters of a mean size of $\langle n\rangle=700-3600$ atoms were found to be stable only on a time scale of days. This instability is attributed to diffusion of atmospheric oxygen into the silica network leading to oxidation of the clusters. The clusters are oxidized successively, the outer layers must first be partially dissolved in the matrix in order to allow penetration of oxygen into the cluster core. 
The use of pre-formed clusters also allowed a confirmation that even clusters embedded inside the matrix and not between silica columns are chemically attacked.

As a consequence the feasibility of silica matrices to stabilize metal clusters must be questioned. While for research purposes it might be possible to measure the optical properties of matrix-isolated clusters in vacuum, this is certainly no alternative in applied physics. Here different approaches must be followed, such as different matrix materials or possibly a protective capping layer preventing diffusion of oxygen into the silica.

\section{Acknowledgments}

The authors gratefully acknowledge fruitful discussions with U. Kreibig and H. Hövel. This work was funded by the Swiss National Science Foundation.

\section{References}

[1] F. Gonella and P. Mazzoldi, Handbook of Nanostructured Materials and Nanotechnology, vol. 4 (Academic Press, CA, 2000).

[2] P. Chakraborty, J. Mater. Sci. 33, 2235 (1998).

[3] M. R. Poulsen, P. I. Borel, J. Fage-Pedersen, J. Hübner, M. Kristensen, J. H. Povlsen, K. Rottwitt, M. Svalgard, and W. Svendsen, Opt. Eng. 42, 2821 (2003).

[4] A. Dhawan and J. F. Muth, Nanotechnology 17, 2504 (2006).

[5] E. Cattaruzza, G. Battaglin, F. Gonella, G. Mattei, P. Mazzoldi, R. Polloni, and B. F. Scremin, App. Surf. Sci. 247, 390 (2005).

[6] U. Kreibig and M. Vollmer, Optical properties of metal clusters, Springer series in materials science (Springer Berlin, 1995).

[7] U. Kreibig, M. Gartz, A. Hilger, and R. Neuendorf, Nanostruc. Mater. 11, 1335 (1999).

[8] C. Félix, C. Sieber, W. Harbich, J. Buttet, I. Rabin, W. Schultze, and G. Ertl, Phys. Rev. Lett. 86, 2992 (2001).

[9] C. Sieber, J. Buttet, W. Harbich, C. Félix, R. Mitrić, and V. Bonačić-Koutecký, Phys. Rev. A 70, 041201 (2004).

[10] T.-H. Lee and R. M. Dickson, Proc. Natl. Acad. Sci. U. S. A. 100, 3043 (2003).

[11] M. Hillenkamp, G. di Domenicantonio, and C. Félix, Rev. Sci. Instrum. 77, 025104 (2006).

[12] S. Serrano-Guisan, G. di Domenicantonio, M. Abid, J.-P. Abid, M. Hillenkamp, L. Gravier, J.-P. Ansermet, and C. Félix, Nature Materials 5, 730 (2006).

[13] H. Haberland, M. Mall, M. Moseler, Y. Qiang, T. Reiners, and Y. Thurner, J. Vac. Sci. Technol. 12, 2925 (1994).

[14] M. A. Garca, M. Garca-Heras, E. Cano, J. M. Bastidas, M. A. Villegas, E. Montero, J. Llopis, C. Sada, G. de Marchi, G. Battaglin, et al., J. Appl. Phys. 96, 3737 (2004).

[15] L. Genzel, T. P. Martin, and U. Kreibig, Z. Phys. B 21, 339 (1975).

[16] S. E. Paje, M. A. Garca, J. Llopis, and M. A. Villegas, J. Non-Cryst. Solids 318, 239 (2003).

[17] H. Hövel, S. Fritz, A. Hilger, U. Kreibig, and M. Vollmer, Phys. Rev. B 48, 18178 (1993).

[18] H. Bi, W. Cai, C. Kan, L. Zhang, D. Martin, and F. Träger, J. Appl. Phys. 92, 7491 (2002).

[19] J. Hu, W. Cai, H. Zeng, C. Li, and F. Sun, J. Phys.: Condens. Matter 18, 5415 (2006).

[20] G. Celep, E. Cottancin, J. Lerme, M. Pellarin, L. Arnaud, J. R. Huntzinger, J. L. Vialle, M. Broyer, B. Palpant, O. Boisron, et al., Physical Review B 70, 165409 (2004).

[21] G. Pacchioni, L. Skuja, and D. Griscom, eds., Defects in SiO ${ }_{2}$ and Related Dielectrics: Science and Technology (Kluwer Academic Publishers, 2000). 
[22] C. J. Brinker and G. W. Scherer, Sol-Gel Science (Academic Press, New York, 1990).

[23] L. Skuja, Journal of Non-Crystalline Solids 239, 16 (1998).

[24] Y. Levy and J. D. Swalen, J. Appl. Phys. 57, 2601 (1985).

[25] J. Lermé, B. Palpant, B. Prével, M. Pellarin, M. Treilleux, J. L. Vialle, A. Perez, and M. Broyer, Phys. Rev. Lett. 80, 5105 (1998).

[26] B. Palpant, L. Saviot, J. Lermé, B. Prével, M. Pellarin, E. Duval, A. Perez, and M. Broyer, Eur. Phys. J. D 9, 585 (1999). 